\title{
Lectura y pensamiento interdisciplinar en las clases de matemáticas y ciencias de la información
}

\author{
Reading and Interdisciplinary Thinking in Mathematics Class and Information Sciences
}

\section{Leitura e pensamento interdisciplinar nas aulas de matemática e ciência da informação}

\author{
Johann Pirela-Morillo' \\ Universidad de La Salle \\ Programa de Sistemas de Información, Bibliotecología y Archivística \\ Bogotá, Colombia \\ jepirela@unisalle.edu.co \\ Wilson Pico-Sánchez ${ }^{2}$ \\ Universidad de La Salle \\ Departamento de Ciencias Básicas. Área de Matemáticas \\ Bogotá, Colombia \\ wilpico@unisalle.edu.co
}

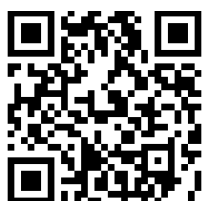

Recibido 16 de setiembre de 2016 • Corregido 4 de agosto de 2017 • Aceptado 24 de agosto de 2017 Received 16 de setiembre de 2016 • Revised 4 de agosto de 2017 • Accepted 24 de agosto de 2017

Recebido 16 de setiembre de 2016 • Revisado 4 de agosto de 2017 • Aprovado 24 de agosto de 2017

Resumen: El objetivo del trabajo es presentar una experiencia docente por medio de la cual se generaron acciones para propiciar el pensamiento interdisciplinar entre las matemáticas y las ciencias de la información. La metodología utilizada se basó en el diseño de estrategias apoyadas en la lectura de dos textos, utilizando como técnica la sistematización didáctica, sustentada en una matriz interdiscursiva como instrumento de registro y análisis de información, en la que se evidenciaron relaciones y significados entre textos. Los resultados revelan la activación de mecanismos para la apropiación compleja e interdisciplinaria del conocimiento y también la concepción de la lectura como herramienta para dominar procesos intelectivos desde un enfoque dinámico. Como conclusión se señala que los ejercicios intertextuales movilizan diversos tipos de saberes para interactuar en los entornos globales de la actualidad.

Palabras claves: Experiencias docentes interdisciplinarias; lectura intertextual; pensamiento interdisciplinar; matemáticas y ciencias de la información.

\footnotetext{
1 Licenciado en Bibliotecología y Archivología, Magíster en Educación. Mención: Currículo, Magíster en Museología, Doctorado y Postdoctorado en Ciencias Humanas. Docente e investigador adscrito al Programa de Sistemas de Información, Bibliotecología y Archivística de la Universidad de La Salle-Colombia.

${ }^{2}$ Licenciado en Matemáticas, Magíster en Ciencias Matemáticas. Docente e investigador adscrito al Departamento de Ciencias Básicas de la Universidad de La Salle-Colombia.
} 
doi: http://dx.doi.org/10.15359/ree.21-3.16

URL: http://www.una.ac.cr/educare

CORREO: educare@una.cr

\begin{abstract}
The objective of the work is to present a teaching experience through which actions were generated to foster interdisciplinary thinking between Mathematics and Information Sciences. The methodology used was based on the design of strategies supported by the reading of two texts, using as a technique the didactic systematization. This systematization was based on an interdiscursive matrix as an instrument for recording and analyzing information, in which relationships and meanings between texts were evidenced. The results revealed the activation of mechanisms for the complex and interdisciplinary appropriation of knowledge, and also the conception of reading as a tool to dominate intellectual processes from a dynamic approach. In conclusion, it is pointed out that intertextual exercises mobilize different types of knowledge to interact in today's global environments.
\end{abstract}

Keywords: Interdisciplinary teaching experiences; intertextual reading; interdisciplinary thinking; mathematics and information sciences.

Resumo: $O$ objetivo deste artigo é apresentar uma experiência educacional através da qual se gero ações para promover o pensamento interdisciplinar entre a matemática e as ciências da informação. A metodologia utilizada baseou-se nas estratégias fundamentadas na leitura de dois textos, usando a técnica da sistematização didática, apoiada em uma matriz interdiscursiva como ferramenta para o registro e análise das informações, nas quais as relações e significados entre palavras foram evidenciadas. Os resultados revelam a ativação de mecanismos de apropriação complexa e interdisciplinar do conhecimento, como também a compressão de leitura como uma ferramenta para dominar os processos intelectuais, partindo de uma abordagem dinâmica. Em conclusão, afirma-se que os exercícios intertextuais movem vários tipos de conhecimento para interagir nos atuais ambientes globais.

Palavras-chave: experiências de educação interdisciplinar; leitura intertextual; pensamento interdisciplinar; matemática e ciência da informação.

\title{
Introducción
}

El pensamiento interdisciplinar se define como el conjunto de operaciones cognitivas y afectivas que permiten el desplazamiento desde los espacios disciplinares, configurado por conceptos y elementos que caracterizan campos específicos del saber, hacia formas mucho más amplias que abarcan las relaciones entre dichos espacios con otros dominios, tal vez lejanos, pero que en esencia pueden conformar tejidos que enriquecen la comprensión de los fenómenos y las realidades que se estudian. La interdisciplinariedad es un nivel de interacción de saberes y de prácticas que remiten a la idea de una cognición estructurada en torno a un entramado de relaciones y discursos, profundizando en la comprensión de la realidad y los fenómenos socioculturales, científicos y tecnológicos.

Transitar hacia un pensamiento interdisciplinar supone la apertura a interacciones entre varios espacios de conocimiento. Con la introducción de estrategias de lectura es posible, desde el aula, crear las condiciones para que el estudiantado realice ejercicios de intersección entre varios campos del saber $y$, con ello, avanzar hacia modelos formativos que superan la visión monolítica del conocimiento, para lograr crear nuevos sistemas de relación con el saber.

Johann Pirela-Morillo y Wilson Pico-Sánchez

Los artículos de la Revista Electrónica Educare del Centro de Investigación y Docencia en Educación de la Universidad Nacional, Costa Rica, se comparten bajo términos de la Licencia Creative Commons: Reconocimiento, № Comercial, Sin Obra Derivada 3.0 Costa Rica. Las autorizaciones adicionales a las aquí delimitadas se pueden obtener en el correo: educare@una.cr 
En este contexto, las instituciones de educación superior deben asumir el reto de desarrollar acciones curriculares y didácticas que fomenten la interdisciplinariedad, como un estado complejo para el establecimiento de conexiones significativas entre diversas disciplinas, con lo cual se configuran, también, posibilidades para el trabajo cooperativo y dialógico que enriquecen los procesos de generación de conocimiento.

En el marco de estas premisas, se presentan experiencias didácticas de lectura desde dos espacios de conocimiento aparentemente lejanos, como es el caso de las matemáticas y las ciencias de la información, con el ánimo de estimular y propiciar en el estudiantado de estas especialidades académicas, la movilización hacia estructuras de pensamiento más abiertas, flexibles y complejas, con lo cual será posible formarlo para que interactúen con mayor eficacia frente a los actuales escenarios turbulentos, impuestos por las lógicas digitales, cuyo sustrato es la virtualidad, la reticularidad, deslocalización y la visión sistémica y holística de la realidad.

Los textos seleccionados para generar los discursos intertextuales fueron, en el caso de las clases de matemáticas: "Caos, fractales y cosas raras" " "El diablo de los números" y de las ciencias de la información, se consideran los textos: "La biblioteca de Babel" y"El desarrollo de colecciones y la selección de recursos en la Biblioteca Universitaria". Los textos se analizan y luego se cotejan, buscando su articulación y relación con los conceptos de: autosimilitud, crecimiento exponencial e infinito, conceptos que también pueden asumirse como metáforas explicativas de las complejas relaciones que se dan en el contexto de las actuales sociedades digitales, en las cuales la incertidumbre, el caos y la sobreabundancia de información son algunas de sus características distintivas.

Como conclusión se destaca la necesidad de incorporar acciones de lectura como estrategias transversales que se pueden incluir en la enseñanza-aprendizaje de diversas asignaturas, relacionadas con diversos campos del saber. La experiencia puso de relieve el potencial de la lectura para activar procesos de desarrollo del pensamiento interdisciplinar, generado estructuras intelectivas que favorecen la interconexión de ideas y de pautas que permiten ampliar los horizontes cognoscitivos, condición esencial para profundizar en la comprensión e interpretación de los fenómenos.

\section{Fundamentos teóricos}

\section{Antecedentes}

En la bibliografía pedagógica y didáctica disponible abundan experiencias mediante las cuales se asume la lectura como estrategia de integración entre diversas disciplinas y como posibilidad concreta de desarrollar procesos del pensamiento. Sin embargo, la mayor parte de estas propuestas han sido formuladas para ser aplicadas en el ámbito de la educación primaria y secundaria, y se evidencian pocos estudios enfocados en la educación superior, de manera puntual en la enseñanzaaprendizaje de campos de conocimiento como las matemáticas y las ciencias de la información: 
doi: http://dx.doi.org/10.15359/ree.21-3.16

URL: http://www.una.ac.cr/educare

CORREO: educare@una.cr

bibliotecología, archivística y áreas conexas. En estos campos del saber se prefieren el uso de didácticas centradas en el desarrollo de procesos cognitivos propios de dichas áreas, tales como el pensamiento lógico formal, la asbtracción, clasificación, categorización, análisis y síntesis.

La experiencia que se desarrolló estuvo orientada hacia el fomento del pensamiento interdisciplinar, para cuya potencialización se precisó, además de los procesos mencionados, otros de mayor nivel de complejidad, como la identificación de pautas a partir de las cuales se pueden conectar dos o más contextos: se trata de los procesos analógicos, de interpretación y argumentación.

Luego de realizar una revisión de investigaciones similares, mediante las cuales se proponen acciones didácticas basadas en la lectura como mecanismo que activa el pensamiento interdisciplinar, se encontraron trabajos en el ámbito colombiano, como el caso de Páez y Hernández (2003), quienes proponen un conjunto de actividades lectoras que promueven la interdiciplinariedad, acudiendo, además, a estrategias para integrar diversas asignaturas en la educación primaria.

Otra experiencia desarrollada en Colombia, que consideramos de gran valor, mediante la cual se aplican estrategias de lectura intertextual fue la llevada adelante por el equipo conformado por Moreno et al. (2009), quienes formularon y ejecutaron el Proyecto literacidades: Una experiencia intra e interdisciplinaria. El proyecto, realizado en la Escuela Normal Superior Nuestra Señora de la Paz ubicada en Bogotá, Colombia; en el barrio Santander, pretendió abordar la lectura de textos, orientada hacia el desarrollo de estructuras cognitivas compatibles con la interdisciplinariedad.

En el ámbito de la educación superior, se aprecia una marcada preocupación por diseñar e investigar sobre acciones didácticas que movilicen saberes y prácticas, partiendo de la mediación y de estrategias intencionadas que procuren alcanzar aprendizajes cada vez más funcionales, significativos y situados. En este sentido, Camacho, Tébar y Díaz (2017) han diseñado un proyecto desde la Vicerrectoría Académica de Universidad de La Salle de Bogotá-Colombia, que pretende contribuir con la reflexión de la propia práctica pedagógica desarrollada por docentes de diversas áreas de conocimiento para aportar en el proceso de cualificación de la docencia universitaria. Una de las premisas consideradas en este proyecto es, precisamente, el hecho de que las investigaciones sobre didácticas para la educación superior son escasas, por lo que se cree que este estudio de sistematización de experiencias podría contribuir a llenar el vacío en materia de reflexiones sobre el hacer docente, desde una perspectiva innovadora e interdisciplinaria.

\section{Lectura intertextual}

Para abordar el proceso de lectura intertextual, como estrategia que permite establecer conexiones entre contenidos presentes en laclase de matemáticas y de ciencias dela información, nos apoyamos en las concepciones sobre intertextualidad y de interdisciplinariedad, en tanto 
que mecanismos mediante los cuales es posible transitar a un desarrollo del pensamiento que proyecta relaciones y sistemas complejos, necesarios para ampliar los horizontes de sentido de la lectura, reconociéndola en su dimensión sistémica y holística.

La lectura intertextual es un ejercicio que permite identificar puntos de encuentro entre dos o más obras literarias o textos, en los cuales es probable que aparentemente no existan conexiones, para lograr, de esta forma, trasladar los significados de los contenidos presentes en un texto, llevándolos a otros textos. En palabras de Bajtín (1982), la intertextualidad se manifiesta en relaciones explícitas e implícitas que contiene una obra literaria con otra obra literaria u otros textos culturales, tales como la pintura, la música, cine, fotografía, poesía, entre otros. En síntesis, la intertextualidad se expresa a partir del nudo o la pauta que puede conectar un significado textual con otro (Noval, 2010).

Siguiendo a Aguilar y Fregoso (2013), diríamos que una de las características de la intertextualidad es la confluencia del significado de un texto anterior con el presente, de manera que se configura una compleja red de relaciones que hacen posible la creación de nuevos sistemas significantes. De esta forma, cuando se aborda la lectura intertextual, el espacio de producción de sentido y de interpretación se extiende, transitando hacia nuevas posibilidades para decodificar, analizar y apropiarse de uno o varios textos. Los autores continúan planteando, fundamentándose en Barthes (1980), que un texto es un intertexto, es decir, una construcción y reconstrucción de escritos que aparecen más o menos reconocibles en él mismo.

Con la teorización de Genette (1989), el concepto de intertextualidad facilita el estudio de los textos. A partir de la visión que se propone, se alude a diversas tipologías de relaciones, denominadas por el autor como trans-textuales, incluidas también la cita sin comillas o con estas, o las referencias tomadas de manera indebida por otros autores o autoras, lo cual, a su vez, implica la posibilidad de que la comprensión de los textos requiere su relación con otros enunciados. Estas relaciones, definidas como trans-textuales aluden al dinamismo que emerge con el hipertexto, concebido como un texto total y envolvente que no se agota desde la propia realidad del texto, sino que se proyecta hacia otras realidades, producidas a partir de contenidos y narrativas diversas, entrecruzadas unas con las otras.

Otro de los elementos que es preciso considerar es la idea de alusión, entendida como fenómeno lingüístico, o más concretamente, semántico. La alusión, entonces, supone visualizar lo dicho sin haber sido expresado, es decir, una referencia indirecta, clara y evidente para los sujetos receptores que escuchan y desconstruyen el texto, en el que bullen universos simbólicos particulares. En la alusión, la transformación del significado es más profunda (Beristáin, 1996). Las alusiones en el texto científico requieren de que el público lector recurra a sus conocimientos previos y del arsenal cognitivo de referencia, con lo cual se imprimirán nuevos sentidos a los conceptos de referencia. 
doi: http://dx.doi.org/10.15359/ree.21-3.16

URL: http://www.una.ac.cr/educare

CORREO: educare@una.cr

\section{Lectura y pensamiento interdisciplinar}

Otras de las estrategias para transitar hacia la expansión de los ámbitos de compresión y de explicación del conocimiento, es la interdisciplinariedad, que se define como una instancia de interacción de los saberes, que permite la creación de escenarios de cooperación y colaboración entre diferentes disciplinas, que a la vez se traducen en formas diversas de aproximarse al mundo (Tamayo, 2004). La interdisciplinariedad significa, ante todo, un cambio de actitud frente a los problemas del conocimiento; es una sustitución de la concepción fraccionada por una unitaria del ser humano y de las realidades que vive.

En esta dirección, si se desea superar la visión atomizada del conocimiento, se requiere repensar el ejercicio docente desde el diseño e implantación de estrategias que respondan a las necesidades del estudiantado y que propendan hacia el desarrollo de un pensamiento interdisciplinar. En este sentido, la lectura que busca generar intersticios temáticos y disciplinares se abre camino como la herramienta que facilita la integración de los saberes, permitiendo la unidad de acciones e interacciones para generar enriquecimiento mutuo de conocimientos. Siguiendo a Mancipe-Flechas (2013), diríamos que se trata de superar la fragmentación disciplinar o la hiper-especialización heredada de la modernidad, avanzando hacia la posibilidad de tender puentes entre las disciplinas.

De esta manera, la lectura debe ser una actividad que se ligue al aprendizaje, a la formación y al conocimiento en el ámbito universitario, porque permite un mayor rendimiento intelectual (Pérez, 2008), ya que promueve la capacidad crítica, la expresión oral y escrita, la argumentación de puntos de vista y la elaboración de ideas conexas con otras disciplinas. Ahora bien, lo que se pretende destacar es la alta potencialidad que tiene la lectura como mediadora para dar el salto cualitativo que permita el desarrollo de estructuras de pensamiento interdisciplinar.

El saber leer es una habilidad que va más allá de descifrar el significado de un mensaje, su alcance está relacionado con acciones como comprender, interpretar, analizar y valorar un texto. Leer críticamente representa la adquisición de "destrezas cognitivas que permitan detectar las intenciones del autor, extraer el contenido que aporta un texto y verificar si es correcto o no" (Cassany, 2006b, p. 82). Si se lee de forma crítica se está en capacidad de dar juicios, es posible ir más allá de lo que se lee, ya que se identifican ideas principales en un texto, se cuenta con argumentos que respaldan proposiciones y se realiza extrapolaciones a otras áreas de conocimiento.

Por otro lado, estudios como los de Creme y Lea (2000), Carlino (2005), Cassany (2006a) y Arnáez (2008) sostienen que cada texto tiene sus particularidades y, en consecuencia, hacen que el ejercicio de leer sea más complejo, desmintiendo con este hecho, que para leer comprensiblemente solo es necesario aprender los principios básicos de la lectura, con lo cual quien lee está en capacidad de aplicarlos a cualquier contexto o situación en los que se requiera. 
Por su parte, Carlino (2005) y Arnáez (2008) sostienen que la tarea de leer involucra a todos los actores del proceso de manera conjunta, y no solo el esfuerzo de quien tiene que hacerlo, dirigirlo o promoverlo. Existe una responsabilidad compartida entre estudiantes, docentes e institución, entes que pueden trabajar de la mano e impulsar estrategias didácticas interdisciplinarias con el fin de dar un tratamiento más integrador al proceso de lectura en la universidad.

Las propuestas anteriores, en particular la de Arnáez (2008), están encaminadas a dar una mirada diferente al proceso de lectura en la universidad y de su importancia para los procesos de comunicación. Se propone que, para generar cultura de lectura y escritura universitaria, se requiere de un compromiso de la institución y de propuestas de índole interdisciplinario. Este es el caso de la Universidad de La Salle, que tiene como política institucional un plan lector, que motiva las interacciones con diferentes áreas del saber. Didáctica enmarcada en el Enfoque formativo Lasallista, EFL (Universidad de La Salle, 2008) y el Canon de los cien libros (Universidad de La Salle, Vicerrectoría Académica, 2012).

Lo anteriormente expuesto nos conduce a enfatizar la necesidad de promover, en las generaciones futuras de profesionales, la lectura interdisciplinar, de modo que puedan emitir juicios, establecer diferencias y comparaciones, y finalmente, estructurar conocimientos para realizar configuraciones organizadas que facilitan los procesos de aprendizaje.

\section{Metodología}

La metodología de trabajo para evidenciar la importancia de la lectura intertextual como ejercicio que potencia el desarrollo del pensamiento interdisciplinar consistió en diseñar experiencias didácticas planificadas para los espacios académicos: Cálculo I del Área de Matemáticas del Departamento de Ciencias Básicas y Desarrollo de Colecciones, perteneciente al Programa de Sistemas de Información y Documentación, ambos de la Universidad de La SalleColombia. La sistematización de experiencias se asumió como método de investigación, el cual, según Gordon de Isaacs (2010), permite la construcción de conocimientos nuevos a partir de la reflexión crítica acerca de las experiencias vividas en proyectos o programas sociales; en este caso, programas de acción didáctica, entendidos como conjunto de actividades para potenciar el pensamiento interdisciplinar.

Con estas experiencias de lectura intertextual se transitó hacia un espacio de convergencia de diversos saberes y prácticas a partir de las cuales se construyen nuevos conocimientos. El ejercicio de intertextualidad se asume como punto de partida para generar un recorrido interdisciplinario entre las matemáticas y las ciencias de la información. 
doi: http://dx.doi.org/10.15359/ree.21-3.16

URL: http://www.una.ac.cr/educare

CORREO: educare@una.cr

\section{Resultados de la experiencia}

En cuanto a la experiencia en el campo de la matemática, la estrategia didáctica aquí presentada es resultado de su integración con diferentes disciplinas a través de la lectura de un libro con contenido matemático. El proceso de comprensión de lectura se realiza mediante la elaboración de un póster de tipo científico desde la transversalidad de las ciencias, a partir de un análisis que considera la matemática, la historia, la ética y el componente social.

La estrategia tuvo como principio que el desarrollo del pensamiento es base para facilitar la incorporación de nuevas ideas o aprendizajes. Como una forma de hacerlo se propone la lectura interdisciplinar, donde el área de matemáticas es el eje articulador que permea las demás disciplinas con conceptos que facilitan la integración de sus saberes. Con lo anterior, se pretende que el estudiantado tenga más elementos para comprender los conceptos abordados en matemáticas y, además, contribuir a la construcción de un currículo integrado.

Este proceso se inició en el año 2013 con la introducción del libro "Caos, fractales y cosas raras" de Braun (2011), donde se buscó que el estudiantado elaborara una serie de preguntas con el contenido del libro, las cuales deberían dar cuenta de sus comprensiones de lectura. Estas debían tener como escenario de formulación las matemáticas y sus relaciones con otras áreas, requiriendo del estudiantado hacer una lectura apoyada en otras fuentes de información y empleando recursos como elaboración de fichas resumen y cuadros sinópticos. Dentro de los conceptos que fueron referentes de estudio estuvieron el de autosimilitud, crecimiento exponencial e infinito, los cuales se asumen como categorías y pautas que conectan los saberes matemáticos y de las ciencias de la información.

Estos conceptos son fundamentales por cuanto constituyen el punto de encuentro para movilizar el pensamiento interdisciplinar, que supera la visión atomizada y de compartimiento estanco del conocimiento, como una entidad monolítica, y lo ubica en la posibilidad de generar diálogos reflexivos que se nutren recíprocamente, dando origen a nuevas pautas de interrelación y comprensión de la realidad.

Por tanto, queriendo que el estudiantado desarrollara habilidades de comprensión de lectura para facilitar el entendimiento de los conceptos matemáticos abordados en el libro propuesto durante el semestre y, al mismo tiempo, realizaran un ejercicio de formación científica, se introduce la estrategia de diseño de un póster científico, el cual debería reflejar las posturas frente a una temática del área de matemáticas.

Además de la lectura sobre los fractales, se consideró el libro:"El diablo de los números. Un libro para todas aquellas personas que temen a las matemáticas" de Enzensberger (2013). A partir de esta historia, el estudiantado se movilizó en búsqueda de información en las diferentes fuentes que les permitiera tener una mejor comprensión del objeto de estudio, el cual finalmente deberían presentar en un evento académico mediante un póster de carácter científico. De los diálogos con colegas, 
surgió la estructura final de la estrategia, ya que el ejercicio de lectura debería contemplar búsqueda de aspectos sociales, culturales, morales, éticos, históricos y matemáticos, los cuales deberían verse reflejados en la construcción del póster científico en torno al concepto de matemáticas elegido.

Finalmente, para la evaluación de la estrategia se elaboró una rúbrica que contemplaba los siguientes criterios: diseño del póster (contar con la estructura de póster científico), imágenes, tablas, texto y ortografía (claridad y estar referenciadas), investigación (referencias bibliográficas y postura clara vista en todos los aspectos), trabajo en equipo (de forma colaborativa) y creatividad y calidad de trabajo (en la ponencia).

Con respecto a la experiencia en el campo de las ciencias de la información, se consideraron también los conceptos medulares definidos como pautas que conectan el área de las matemáticas con este campo, el cual, por cierto, tiene un carácter y esencia interdisciplinaria, debido a que se relaciona con los diversos ámbitos de las ciencias. Tales conceptos son: autosimilitud, exponencialidad e infinito. La intertextualidad se evidenció a partir de la relación entre el texto de Borges (1974) y Negrete (2003), referido este último al desarrollo de colecciones en bibliotecas.

La experiencia docente consistió en diseñar y ejecutar un ejercicio de lectura intertextual entre el cuento: "La Biblioteca de Babel" del escritor argentino Borges (1974) y el libro del canon del espacio académico: Desarrollo de colecciones, titulado: El desarrollo de colecciones y la selección de recursos en la biblioteca universitaria, de Negrete (2003). El texto de Borges (1974) es utilizado como metáfora para explicar complejidades que surgen en el ciberespacio, algunas de las cuales tienen que ver con la interconexión, dinamismo y ubicuidad de la información.

La lectura intertextual se justificó por la necesidad que se plantea en los actuales entornos signados por la globalidad y la deslocalización, de recurrir a la relación de los discursos expuestos en ambos textos, logrando identificar pautas de relación entre lo expresado por el escritor argentino como precursor del ciberespacio, desde la literatura, y lo propuesto por Negrete (2003), en relación con la necesidad de abordar los procesos de desarrollo de colecciones como un conjunto de estrategias que conducen al fortalecimiento de los recursos reunidos en las instituciones bibliotecarias para responder a las necesidades del público usuario.

Luego de aplicar las estrategias de lectura intertextual para cada campo del conocimiento: matemáticas y ciencias de la información, se logró evidenciar el potencial que existe en los textos estudiados: "Caos, fractales y cosas raras" de Braun (2011), "El diablo de los números" de Enzensberger (2013) "La Biblioteca de Babel" de Borges (1974) y “El desarrollo de colecciones y la selección de recursos en la biblioteca universitaria" de Negrete (2003), es posible plantear que desde las lecturas realizadas se pueden activar procesos de pensamiento interdisciplinar, a partir de los conceptos ya señalados: autosimilitud, exponencialidad e infinito, en el entendido de que tales conceptos pueden ser asumidos como categorías o metáforas que permiten explicar las relaciones con el conocimiento en el contexto de la sociedad digital, en la cual se ponen de relieve estos conceptos (Ver Tabla 1). 
doi: http://dx.doi.org/10.15359/ree.21-3.16

URL: http://www.una.ac.cr/educare

CORREO: educare@una.cr

Tabla 1: Matriz de lectura intertextual

\begin{tabular}{|c|c|c|c|c|}
\hline \multirow[b]{2}{*}{ Categorías } & \multicolumn{2}{|c|}{ Matemáticas } & \multicolumn{2}{|c|}{ Ciencias de la Información } \\
\hline & $\begin{array}{l}\text { "Caos, fractales y } \\
\text { cosas raras" }\end{array}$ & $\begin{array}{l}\text { "El diablo de los } \\
\text { números" }\end{array}$ & $\begin{array}{l}\text { "La biblioteca de } \\
\text { Babel" }\end{array}$ & $\begin{array}{l}\text { "El desarrollo de colecciones y } \\
\text { la selección de recursos en la } \\
\text { biblioteca universitaria" }\end{array}$ \\
\hline Autosimilitud & $\begin{array}{l}\text { "Las famosas muñecas rusas } \\
\text { están formadas por una mu- } \\
\text { ñeca grande en cuyo interior } \\
\text { se halla otra muñeca, similar } \\
\text { a la grande, pero de menor } \\
\text { tamaño. Dentro de esta se- } \\
\text { gunda muñeca, ... similar [a } \\
\text { la grande,] pero de tamaño } \\
\text { aún menor. Un conjunto con- } \\
\text { tiene cinco o seis muñecas, } \\
\text { todas similares, pero cada } \\
\text { una de menores dimensio- } \\
\text { nes" (Braun, 2011, p. 31). }\end{array}$ & $\begin{array}{l}\text { "Estrellas de seis puntas } \\
\text { dentro de una estrella de } \\
\text { seis puntas, rayos que se ra- } \\
\text { mificaban en rayos cada vez } \\
\text { más pequeños... -¿No son } \\
\text { maravillosos estos copos?" } \\
\text { (Enzensberger, 2013, pp. } \\
\text { 189-190). }\end{array}$ & $\begin{array}{l}\text { "Como todos los hombres } \\
\text { de la Biblioteca, he } \\
\text { viajado en mi juventud; } \\
\text { he peregrinado en busca } \\
\text { de un libro, acaso del } \\
\text { catálogo de catálogos" } \\
\text { (Borges, 1974, p. 465). }\end{array}$ & $\begin{array}{l}\text { “El desarrollo de colecciones... } \\
\text { implica un estudio destinado } \\
\text { a incrementar, mantener y } \\
\text { actualizar las colecciones... } \\
\text { para que respondan a las } \\
\text { necesidades de investigación } \\
\text { y de enseñanza de los usuarios } \\
\text { específicos, lo que implica: ... } \\
\text { estudiar las necesidades de los } \\
\text { usuarios" (Negrete, 2003, p. 24). }\end{array}$ \\
\hline $\begin{array}{l}\text { Crecimiento } \\
\text { exponencial }\end{array}$ & $\begin{array}{l}\text { "Cada vez que pasamos de } \\
\text { una figura a otra la longitud } \\
\text { total va disminuyendo, } \\
\text { pero el número de líneas va } \\
\text { aumentando (de } 1 \text { a } 2 \text { a } 4 \text { a } \\
8 \text { a } 16 \text { a } 32 \text { a...). Si así conti- } \\
\text { nuáramos indefinidamente, } \\
\text { el número de líneas crecería } \\
\text { sin límite y..." (Braun, 2011, } \\
\text { pp. 96-97). }\end{array}$ & $\begin{array}{l}\text { "Si haces saltar el diez una } \\
\text { vez se queda como está: } 10^{1} \\
=10 ; \text { Si lo haces saltar dos: } \\
10^{2}=100 ; \text { Si lo haces saltar } \\
\text { tres } 10^{3}=1000 ;- \text { si lo hago } \\
\text { saltar cinco veces -exclamó } \\
\text { Robert-, da 100.000. Otra } \\
\text { vez, y me sale un millón. - } \\
\text { Hasta el aburrimiento -dijo } \\
\text { el diablo de los números-" } \\
\text { (Enzensberger, 2013, p. 41). }\end{array}$ & $\begin{array}{l}\text { "La biblioteca es total..., } \\
\text { las autobiografías de los } \\
\text { arcángeles, el catálogo } \\
\text { fiel de la biblioteca, miles } \\
\text { y miles de catálogos... } \\
\text { el comentario de ese } \\
\text { evangelio, el comentario } \\
\text { del comentario de ese } \\
\text { evangelio" (Borges, 1974, } \\
\text { pp. 467-468). }\end{array}$ & $\begin{array}{l}\text { “El desarrollo de colecciones } \\
\text { es el proceso que permite } \\
\text { satisfacer las necesidades y } \\
\text { demandas de información } \\
\text { mediante la formación } \\
\text { de colecciones básicas y } \\
\text { fortalecidas, tanto en alcance } \\
\text { como en profundidad en todas } \\
\text { las áreas y temas de interés... y } \\
\text { puede complementarse con el } \\
\text { acceso... de aquellos recursos } \\
\text { que se localizan fuera de la } \\
\text { biblioteca” (Negrete, 2003, p. 25). }\end{array}$ \\
\hline Infinito & $\begin{array}{l}\text { "Una consecuencia de la } \\
\text { aplicación de esta función es } \\
\text { que, al transcurrir el tiempo, } \\
\text { la población crecerá de } \\
\text { [forma] indefinida; llegará } \\
\text { un momento en [el] que [la } \\
\text { población] será tan grande } \\
\text { que el número de individuos } \\
\text { de la especie no cabría en el } \\
\text { planeta” (Braun, 2011, p. 41). }\end{array}$ & $\begin{array}{l}\text { “Le asombró cuánta gente } \\
\text { cabía entre la puerta y la } \\
\text { cama. Los números pasaban } \\
\text { ante él como ciclistas de } \\
\text { competición o corredores } \\
\text { de maratón, porque todos } \\
\text { llevaban sus números en } \\
\text { camisetas blancas. El cuarto } \\
\text { era bastante pequeño, } \\
\text { pero cuantos más números } \\
\text { se apretujaban más largo } \\
\text { parecía. La puerta se fue } \\
\text { alejando cada vez más, has- } \\
\text { ta que apenas fue posible } \\
\text { distinguirla al final de un } \\
\text { recto pasillo" (Enzensberger, } \\
\text { 2013, pp. 170-171). }\end{array}$ & $\begin{array}{l}\text { "El universo (que otros } \\
\text { Ilaman biblioteca) se } \\
\text { compone de un número } \\
\text { indefinido, y tal vez } \\
\text { infinito, de galerías } \\
\text { hexagonales, con vastos } \\
\text { pozos de ventilación en } \\
\text { el medio, cercados por } \\
\text { barandas bajísimas. Des- } \\
\text { de cualquier hexágono } \\
\text { se ven los pisos inferiores } \\
\text { y superiores: intermina- } \\
\text { blemente" (Borges, 1974, } \\
\text { p. 465). }\end{array}$ & $\begin{array}{l}\text { “El concepto de desarrollo de } \\
\text { colecciones enfatiza no solo la } \\
\text { responsabilidad que tiene la } \\
\text { biblioteca para utilizar juicio } \\
\text { crítico al construir colecciones } \\
\text { de calidad, sino también la } \\
\text { necesidad de llevar a cabo una } \\
\text { adecuada administración de la } \\
\text { calidad, cantidad y equilibrio } \\
\text { temático" (Negrete, 2003, p. 26). }\end{array}$ \\
\hline
\end{tabular}

Nota: Elaboración propia.

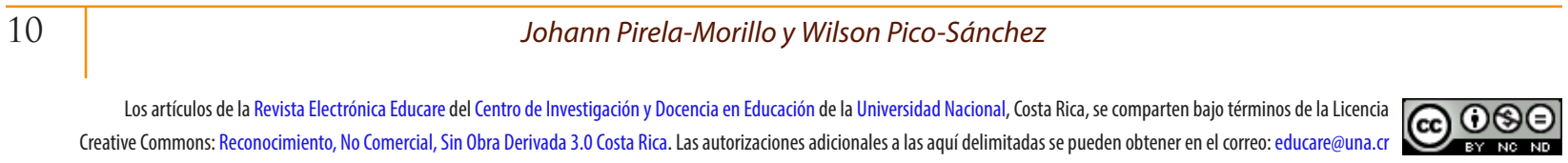


Como puede observarse en la matriz intertextual, las pautas que conectan los textos son los conceptos de: autosimilitud, crecimiento exponencial e infinito. Las ideas expuestas en los cuatro textos analizados y cotejados ponen de relieve, en primer lugar, que la autosimilitud se expresa en la idea subyacente las muñecas rusas, que contiene una similar, pero de menor tamaño dentro de la otra. Esta metáfora de lo autocontenible también se puede relacionar con la idea de catálogos de catálogos en el caso de la Biblioteca de Babel de Borges (1974), noción que a su vez se asocia con la posibilidad de encontrar similitudes, mediante la comparación de la información disponible en catálogos, con lo cual se introduce también el concepto de complejidad y de relación arborescente con los datos y con el conocimiento en el contexto de la sociedad digital.

El crecimiento exponencial se asume como pauta para la interconexión textual e interdisciplinar. En este sentido, la exponencialidad alude a una idea de crecimiento sin límites, como es el caso del mundo virtual que se ha venido configurando con las redes de información y las redes sociales, en las cuales la relación con el conocimiento se proyecta en espacios de sobre abundancia de contenidos, disponibles en sitios, portales, blogs y demás espacios de la trama digital. Esta exponencialidad es expresada en los cuatro textos cotejados como una característica típica que introduce lógicas cada vez más dinámicas e interactivas, cuyo sustrato es el incremento a escala ilimitada.

Por último, el concepto de infinito complementa las dos nociones anteriores, y se ve de manera concreta en los textos estudiados, mediante registros que señalan lo indefinido, como el inventario interminable de situaciones y procesos. Lo infinito como característica presente en las realidades digitales, construidas a partir de universos informacionales y comunicacionales: los números son infinitos y también lo es el mundo borgesiano de la biblioteca compuesta por galerías hexagonales, en las cuales bullen saberes diversos, estructurados a su vez sobre la base de ideas y de información susceptible de interconectarse una con otra.

\section{Conclusiones}

Luego de abordar un ejercicio que permitió establecer posibilidades reales de interdisciplinariedad, a partir de la lectura en dos ámbitos de conocimientos aparentemente lejanos como el de las matemáticas y el de las ciencias de la información, es posible recapitular sobre los siguientes elementos que permiten al mismo tiempo proyectar algunas conclusiones provisionales.

El desarrollo de la estrategia de intertextualidad entre dos campos colocó de relieve la posibilidad de encontrar registros que conectan cuatro textos, en cuyos argumentos se observó la presencia subyacente de conceptos-categorías como: autosimilitud, crecimiento exponencial e infinito, las cuales constituyen patrones que permiten profundizar en la comprensión de 
doi: http://dx.doi.org/10.15359/ree.21-3.16

URL: http://www.una.ac.cr/educare

CORREO: educare@una.cr

la complejidad inherente a los fenómenos que se viven actualmente, con las denominadas sociedades globales de la información y del conocimiento, para cuya interacción efectiva se requiere, en primer lugar, acercarse a la naturaleza y alcance de su estructura y configuración. De esta forma, se logró aprovechar las clases de matemáticas y de ciencias de la información como pretextos, para construir intertextos que permitan explicar las claves esenciales de este tipo de sociedades, signadas por estructuras cada vez más por metáforas conceptuales que se encuentran en las obras trabajadas.

La sistematización de experiencias docentes permitió también evidenciar que la interdisciplinariedad es posible potenciarla, mediante procesos de lectura intertextual, entendida como práctica reflexiva e interdiscursiva, cuyo sustrato es el pensamiento analítico, sintético y analógico, construido por medio del encuentro entre dos o más obras literarias o textos, y se logra, de esta forma, trasladar significados de los contenidos presentes en un texto hacia otros textos o contextos del pasado o del presente.

Para la construcción de la trama intertextual, es necesario recurrir primero a la identificación de pautas de conexión, desde las cuales se realicen ejercicios interpretativos. Tales pautas que conectaron los encuentros intertextuales fueron los conceptos de: autosimilitud, crecimiento exponencial y autosimilitud, como referentes y metáforas que permitieron tejer relaciones intertextuales e interdisciplinares entre los campos de conocimiento mencionados. Lo interdisciplinario se asumió como una instancia de interacción de los saberes, que permite la creación de escenarios de cooperación y colaboración entre diferentes disciplinas, que a la vez se traducen en formas diversas de aproximarse al mundo, lo cual implica transitar desde una concepción fraccionada y parcelada del conocimiento a la idea de puentes por una unitaria de los actores sociales y de las realidades que viven.

Las experiencias didácticas expuestas desde la clase de matemáticas y de ciencias de la información revelan la alta potencialidad de la lectura como mediadora en los procesos de desarrollo del pensamiento interdisciplinar, y abren la posibilidad de indagar alternativas diversas que amplían la comprensión de los textos y permiten acceder a otras formas para entender las realidades complejas de las actuales sociedades.

\section{Referencias}

Aguilar, L. Ey Fregoso, G. (2013). La lectura de la polifonía e intertextualidad en el texto científico. Revista mexicana de investigación educativa, 18(57), 413-435. Recuperado de http://www. redalyc.org/pdf/140/14025774005.pdf

Arnáez, P. (2008). Leer y escribir en la universidad: Una propuesta interdisciplinar. Enunciación, 13(1), 7-19. Recuperado de http://revistas.udistrital.edu.co/ojs/index.php/enunc/article/ view/1256/1690 
Bajtín, M. (1982). Estética de la creación verbal. México: Siglo XXI.

Barthes, R. (1980). S/Z. México: Siglo XX.

Beristáin, H. (1996). Alusión, referencialidad, intertextualidad (Vol. 1). México: UNAM.

Borges, J. L. (1974). La biblioteca de Babel. En C. V. Frías (Ed.), Jorge Luis Borges obras completas 123-1972 (pp. 465-471). Buenos Aires: Emecé Editores. Recuperado de https://literaturaargentina1unrn.files.wordpress.com/2012/04/borges-jorge-luis-obrascompletas.pdf

Braun, E. (2011). Caos, fractales y cosas raras. México: Fondo de Cultura Económica.

Camacho, C. A., Tébar, L. y Díaz, M. (2017). Proyecto: Innovación didáctica para la educación superior. Bogotá-Colombia: Universidad de La Salle, Vicerrectoría Académica.

Carlino, P. (2005). Escribir, leer y aprender en la universidad. Una introducción a la alfabetización académica. Buenos Aires: Fondo de Cultura Económica.

Cassany, D. (2006a). Taller de textos. Leer, escribir y comentar en el aula. Barcelona: Paidós.

Cassany, D. (2006b). Tras las líneas. Sobre la lectura contemporánea. Barcelona: Anagrama.

Creme, P. y Lea, M. R. (2000). Escribir en la universidad. Barcelona. Gedisa.

Enzensberger, H. M. (2013). El diablo de los números. Un libro para todos aquellos que temen a las matemáticas. Madrid: Siruela.

Genette, G. (1989). Palimpsestos: La literatura en segundo grado. Madrid: Taurus.

Mancipe-Flechas, E. (2013). El tránsito de la disciplinariedad a la transdisciplinariedad. En L. E. Pérez (Ed.), Pensar en escuelas de pensamiento. Una aproximación interdisciplinar y transdisciplinar (pp. 30-42). Bogotá: Ediciones Unisalle.

Moreno, P. J., Lamprea, J. A., Bastidas, M. A. y González, J. M. (2009) Literacidades: Una experiencia intra e interdisciplinar. En A.Velasco (Ed.), La lecturay la escritura como procesos transversales en la escuela. Experiencias innovadoras en Bogotá (Capítulo 17, pp. 263-272). Recuperado de http://cms.univalle.edu.co/todosaaprender/anexos/lugaresdestacados/10-Lecturayes crituracomoprocesostransversales.pdf

Negrete, M. (2003). El desarrollo de coleccionesy la selección de recursosen la biblioteca universitaria. México: UNAM.

Noval, C. (2010). La polifonía y la intertextualidad en producciones textuales infantiles. Cuadernos de Lingüistica Hispánica, 15, 139-150. Recuperado de http://www.redalyc.org/articulo. oa?id=322227521009 
doi: http://dx.doi.org/10.15359/ree.21-3.16

URL: http://www.una.ac.cr/educare

CORREO: educare@una.cr

Páez, J. M y Hernández, A. (2003). La lectura eje interdisciplinario como herramienta pedagógica (Tesis de licenciatura). Universidad de la Sabana, Bogotá, Chía. Recuperado de https:// intellectum.unisabana.edu.co/bitstream/handle/10818/5799/128309.PDF?sequence $=1$

Pérez, J. (Ed.). (2008). Palabras por la lectura. Toledo: Consejería de Cultura de la Junta de CastillaLa Mancha.

Tamayo, M. (2004). Diccionario de la investigación científica (2a ed.). México: Limusa.

Universidad de La Salle. (2008). Enfoque formativo lasallista EFL. Bogotá: Autor. Recuperado de $\quad$ http://www.lasalle.edu.co/wps/wcm/connect/e2b72d85-7970-4361-a8b2c8026ce6b53c/28-enfoque-formativo-lasallista-efl.pdf?MOD=AJPERES

Universidad de La Salle, Vicerrectoría Académica. (2012). Canon de los 100 libros Una estrategia de lectura hacia su consolidación (Colección Hitos N. 15). Bogotá: Autor. 